\title{
Modeling of Microtubule Structure and Dynamics: Concepts and Mechanism of Reactions Inside a Microtubule
}

\author{
Maliheh AkbarMoulaee ${ }^{1}$, Majid Monajjemi ${ }^{2, * D}$, Jamshid Mehrzad ${ }^{1}$, Alireza Motavalizadehkakhky ${ }^{3}$, \\ Mitra Naeimi ${ }^{4}$ \\ Department of Biochemistry, Neyshabur Branch, Islamic Azad University, Neyshabur, Iran \\ Department of Chemical engineering, Central Tehran Branch, Islamic Azad University, Tehran, Iran \\ Department of Chemistry, Neyshabur Branch, Islamic Azad University, Neyshabur, Iran \\ Department of Biomedical Engineering, Central Tehran Branch, Islamic Azad University, Tehran, Iran \\ Correspondence: maj.monajjemi@iauctb.ac.ir;
}

Scopus Author ID 6701810683

Received: 18.08.2020; Revised: 10.09.2020; Accepted: 11.09.2020; Published: 13.09.2020

\begin{abstract}
Via docking energy and interactions inside microtubules, TXL and stathmin molecules have been studied, and it has been shown that better binding capabilities in TXL are related to the environment of neighbor amino acids compared to other toxoids. Molecular dynamics simulation of the best-docked complexes of several ligands has been done for presenting the new concepts and mechanism of reactions inside a microtubule complex
\end{abstract}

Keywords: microtubule; stathmin; Taxol; docking simulation.

(C) 2020 by the authors. This article is an open-access article distributed under the terms and conditions of the Creative Commons Attribution (CC BY) license (https://creativecommons.org/licenses/by/4.0/).

\section{Introduction}

Microtubules are long protein filamentous structures that form of the dynamic cytoskeletal with morphological changes and have multiple functions inside the cellular processes such as peculiar biophysical settings in the internal environment. The microtubule structure is similar to the tubular polymers, while its thickness diameter is about $25 \mathrm{~nm}$ in which the heterodimers are assembled head to tail in a polar fashion [1-5]. The "building brick" from which they are formed called $\alpha \& \beta$ tubulins with a protein structure and appears in solution as a dimer of two similar subunits, and a variety of proteins can be attached, in which the microtubule-associated proteins (MAPs) can play a more structural role (MAP1, MAP2, tau, etc.). Although many of MTs are largely excluded from the cytoplasmic influence, $2.5 \mathrm{~nm}^{2}$ lateral pores and $205 \mathrm{~nm}^{2}$ entrances at its ends are connected to the cytoplasm. Recently, especial and spherical particles have been explored in their structures that the particles' existence among cell types while the neuronal cells have the most particles. Although identification of components inside of luminal has been difficult, during the next 25 years, observation of those particles in the microtubules lumens has culminated using vitreous cryoelectron microscopy $[4,6]$. Those proteins make microtubule turnover in cells, contributing to the microtubule cytoskeleton. Thus, a detailed review of its structure can be considered the tubulin, motor proteins, and their effects on its function, including dynamics of assembly, transport mechanisms, interactions with other cell components [5,7]. Stathmin families were initially recognized as a protein to respond to the extracellular signals in highly proliferative breast cancers and malignant ovarian cancers. Stathmin is an inhibitor that makes a tight 
interaction with related tubulins. One of the major stathmin proteins is named $\mathrm{RB}_{3}$, which shares with other groups of the stathmin-like domain (SLD). It has a disordered structure, and its activity is down-regulated through multiple phosphorylations. Stathmin is a soluble, cytoplasmic protein that enforces an important role in regulating rapid microtubules reconstructing of the cytoskeleton due to the cell's requirements. At low concentrations of free tubulins, both alpha and beta in the cytoplasm, the growth rate at the microtubule ends is languid and results in an increased rate of disassembly or de-polymerization [8,9].

\subsection{GTP and microtubule end.}

Non-polymerized tubulins exist as a heterodimer of " $\alpha$-tubulin" \& " $\beta$-tubulin" with binding sites for two molecules of guanosine triphosphate (GTP), one exchangeable and the other not. Via electron microscopy (EM) it can be seen that each proto-filament consists of globular $4 \mathrm{~nm}$ subunits, and it is possible for refined tubulin to assemble with a range of diameters containing between 9 and 16 proto-filaments. The building blocks of microtubules are arranged in a polar fashion along the proto-filaments. In other words, microtubules as a whole are polar and usually distal to nucleating centers [5-8]. In addition, the more dynamic than the minus end that is attached to nucleation centers (Fig.1-a). Another property of tubulins is that each subunit can bind GTP. The GTP bound to alpha-tubulin appears to have a structural role and cannot be exchanged. On the other hand, the GTP attached to beta-tubulin can be exchanged against GTP from the solution. It is the beta-tubulin associated GTP that is hydrolyzed to GDP during microtubule assembly so that the beta-tubulin in the interior of a microtubule contains GDP. This hydrolysis provides the driving force for microtubule dynamics (Fig. 1-b) [10,11]. Two atomic structures of pair tubulins up to now are known that. The first one is GDP-tubulin in straight antiparallel proto-filaments of two-dimensional zinc sheets stabilized by Taxol (Fig. 2) that has been employed to construct pseudo-atomic models of microtubules. As can be seen in Fig. 2, the GTP is sandwiched between $\alpha$ and $\beta$ tubulin subunits of each heterodimer. The nucleotide bound to $\beta$-tubulin has been hydrolyzed to GDP through contact with helix H8 and loop T7 of the activation domain of another $\alpha$-tubulin subunit. Taxol sits in the pocket of $\beta$-tubulin on the inside face of microtubules. In beta-tubulin, this pocket is occupied by the extended L-loop. The second is the curved structure of two head to tail GDP-tubulin dimers in complex with the stathmin-like domain of RB3 (RB3-SLD) [1214]. The structure of tubulin in microtubules is related to the straight zinc-sheet structure since both are made of similar proto-filaments, although the different lateral contacts cause modifications. Regarding polarity, a natural interpretation was that the terminal subunits on the plus end are beta-tubulin in association with bound GTP (Fig. 1b). However, another possibility is the inverted arrangement (Fig. 1C) where the terminal layer is alpha-tubulin, with betatubulin buried inside but still retaining its bound GTP [10-14]. This would imply that the terminal crown at the minus end is beta-tubulin (Fig. 1C). Image reconstruction of microtubules decorated with kinesin now suggests that the polarity of Fig. 1C is the correct one, that is, alpha-tubulin at the plus end and beta-tubulin at the minus end. This can be supposed a higher dynamic activity might be observed at the plus end, but it could presumably be explained if a high-resolution structure of tubulin were available. As a microtubule is polar, the plus end is usually distal, the minus end proximal to a microtubule nucleating center. The exchangeable GTP site is on beta-tubulin; this GTP becomes hydrolyzed to GDP upon the incorporation of the subunit into a microtubule (Fig. 1). This figure emphasizes two points: first, that the plus end terminates with a crown of alpha subunits that implies the minus end. Second, microtubules 
can be decorated with one kinesin head per tubulin dimer, bound to beta-tubulin. The axial distance between kinesin heads is $8 \mathrm{~nm}$, consistent with the length of a dimer $[15,16]$.

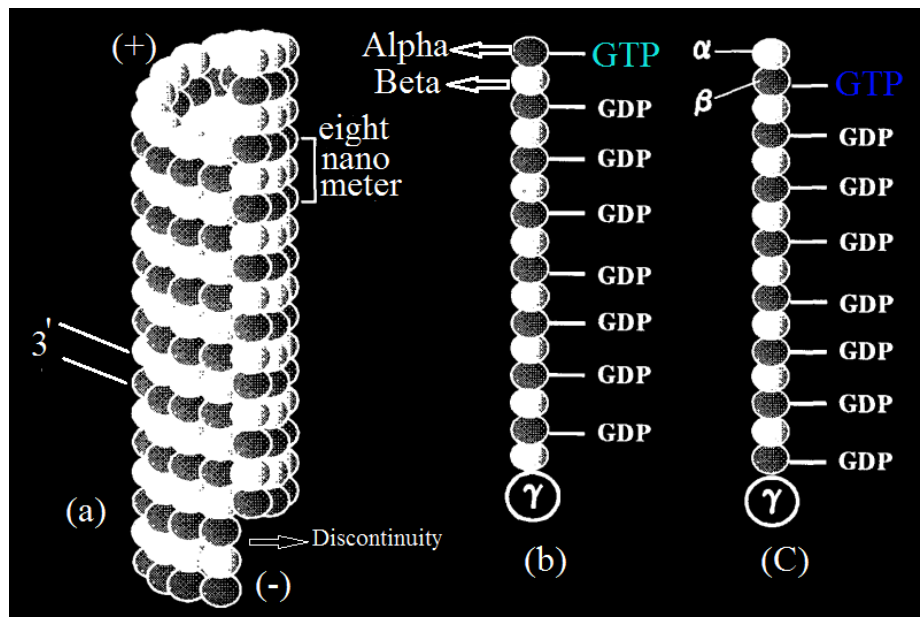

Figure 1. (a) The hollow cylinder consists of 13 protofilaments, each consisting of a string of alternating tubulin subunits. (b) and (c) Alternative models of protofilament polarity. In both cases, the microtubule-bound GTP is on the plus end, whereas the interior subunits contain GDP

In Fig. 1 (b \& c), in both cases, the microtubule-bound GTP is on the plus end, whereas the interior subunits contain GDP. On the minus end, a subunit of gamma-tubulin as this is involved in nucleating microtubules off the microtubule-organizing centers. Adjacent monomers in proto-filaments arrange a series of helices that for 13-protofilament microtubule 3 helices run in parallel, and for a smaller or larger number of proto-filaments, there may be two or four helices [17-20].

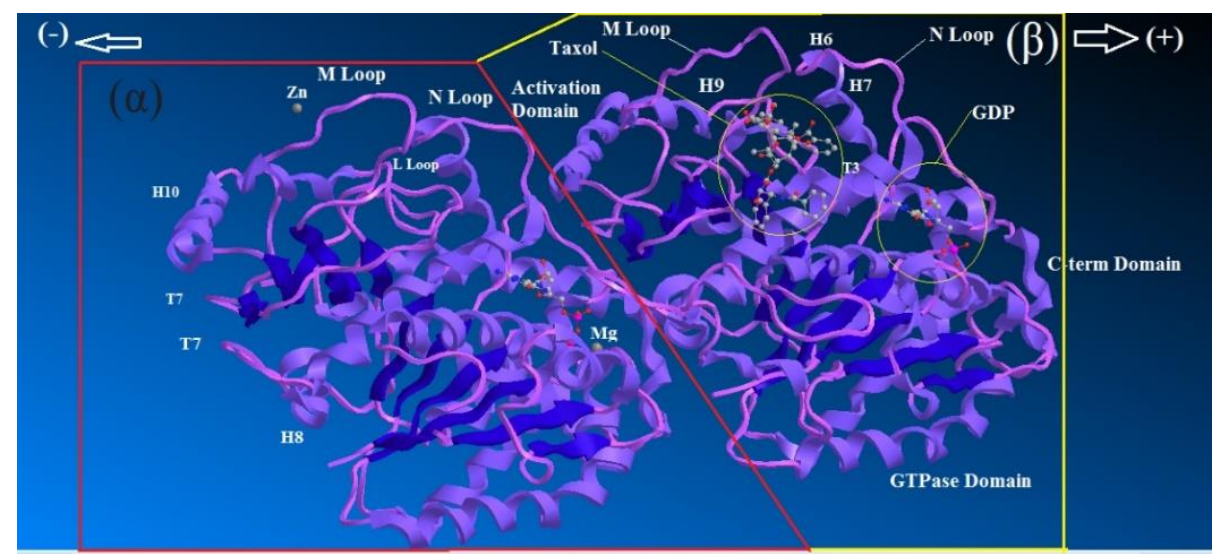

Figure 2. Ribbon diagram of $\alpha \beta$-tubulin heterodimer including taxol, GTPase domain, the activation domains, the core helix that connects the two globular domains in each monomer, and the C-terminal domain on the external surface is shown.

These differences prepare several flexibilities in their bonds among adjacent heterodimers. Microtubules-associated motor proteins, such as dynein and kinesin, can be run for long distances along a microtubule when there are 13 proto-filaments.

\subsection{Microtubule surface lattice.}

There are two type lattices for microtubules, including the 'A'" $\mathrm{A}$ ' lattice for the Amicrotubules of outer flagellar doublets and the 'B' lattice for B-tubules. Both lattices have the same polar arrangement of proto-filaments, but they differ in the stagger between proto- 
filaments; the A-lattice has a stagger of about $3.0 \mathrm{~nm}$, the B-lattice has about $0.95 \mathrm{~nm}$. The Blattice requires some kind of discontinuity that this situation has now changed due to the contrast between alpha and beta -tubulins where can be extremely increased by labeling betatubulin of the motor protein, kinesin. Chemical crosslinking has exhibited that the kinesin head is attached to beta-tubulin, implying a stoichiometry of one head per tubulin dimer. Therefore, the axial spacing of the kinesin head along microtubules is 8 Nanometer, which corresponds to the tubulin dimer spacing. X-ray diffraction analysis has also exhibited that the lattice of dimers is B-type. In cryoelectron microscopy, the head appears to form an angle which has an $8 \mathrm{~nm}$ axial spacing, as well[16-20].

\subsection{Stathmin-microtubules interactions.}

Stathmin interacts with alpha and beta -tubulins for forming a ternary compound with 1:2 mole ratio (one mole of stathmin binds to two moles of tubulin dimers) through the SLD. Tubulins are able to replace among curved structure with the domain of the $\mathrm{RB}^{3}$ protein and a straight microtubule-like structure (Fig. 3). The disassembly has done through GTP hydrolysis, and GTP would allosterically induce a straight conformation of tubulins during microtubule assembly, and also GDP would induce a curved conformation approving disassembly.

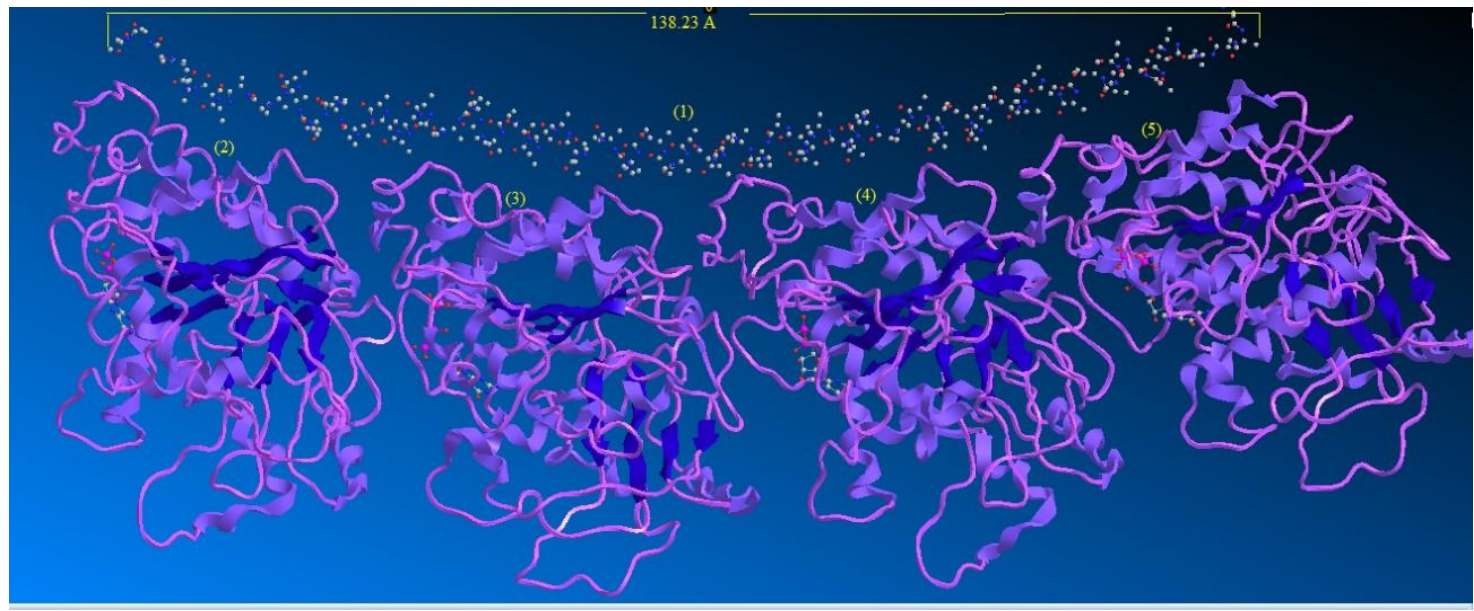

Figure 3. Interaction of stathmin with alpha and beta tubulins (4 subunits).

Microtubule-associated protein or MAPs of tubulin-GDP complexes have confirmed different inter-dimer curvatures, and this kind mechanism is confirmed by the GTP-bound $\gamma$ tubulin concept. This confirmation led to a suitable situation that the free GTP-tubulin dimers are driven into the straight configuration via the microtubule. Therefore the GTP $\gamma$-phosphate only lowers the unfavorable free energy differences among the curved and the straight structure. As a result, GTP binding might be induced a straighter position in the solution for lateral interactions in the lattice model. It might be important for the understanding of the GTP hydrolysis mechanism for destabilizing the microtubule lattice. Although these understanding predict the GTP hydrolysis mechanism into the lattice, how this strain affects the strength of longitudinal and lateral bonds to destabilize the microtubule remains unknown [15-19].

\subsection{Reactions inside microtubules.}

Currently, researchers believe that the interior of microtubules can be more dynamic in contrast to previously thought. This might be preparing a straight route for the redistribution of microtubule lengths during the restructuring of the cell, such as appears during mitosis, rather 
than relying just on the higher degree of dynamic instability that also accompanies this process. Second advantage of internal breakage is that it greatly speeds up microtubule disassembly. In some marine organisms, this takes place in preformed regions and in a $\mathrm{Ca}^{2+}$ dependent fashion. It is notable using cryo-electron-microscopy can be exhibit lattice defects where the protofilament number changed abruptly within microtubules. In addition, capped microtubules at both ends by flagellar axonemal and also the remaining tubulin out demonstrated that these microtubules are very stable, except for the cases where a breakpoint developed inside. Meanwhile, it might remember that proto-filaments of disassembling microtubules can coil and fray apart from the end to points deep inside the microtubule, and even at bends inside a microtubule. In this way, the bending, breaking, or severing appears both in vitro and in vivo and will start through nicking the wall among those proto-filaments.

A method to stiffen microtubules is to pack them into bundles that this process can be enhanced via transfecting cells with cDNAs for MAPs such as MAP2, MAP2c, or tau (Fig. 2). These MAP proteins have homologous microtubule-binding domains but differ greatly in the size of the amino-terminal 'projection' domain. The dominant effect on the spacing between microtubules in a bundle appears to be the size of the projection domain; for instance, MAP2 keeps microtubules further apart than tau. Whether or not these MAPs actually crosslink microtubules at a given spacing is another issue. This depends possibly on the nucleation activity of MAPs, but not necessarily on their crosslinking activity [18-20].

\section{Materials and Methods.}

\subsection{Modeling of microtubule structure and dynamics.}

In self-assembling biopolymers, one of the major things is to understand how the proper structure is initiated or started and also how it then grows via the addition of domains and elongation. Microtubules demonstrate additional trouble due to they are not stable, even after assembling that has reached an overall steady state. These kind phenomena are known as dynamic instability, while the transitions among growth and shrinkage are catastrophe and "rescue", respectively.

There are general agreements that these kind instabilities are dependent on GTP hydrolysis, whereby irreversible chemical reactions are coupled to microtubule assembling, but they in themselves do not suffice for explaining that phenomenon. Several recent papers have addressed different aspects of elaborated the lateral cap model, which suppose that microtubule dynamics are determined through the terminal subunits and their immediate environment.

While the previous formulation of the model was based on the lattice, the recent behaviors are lattice-independent and thus more usual. It has been considered the problem of how growing and shrinking microtubules can coexist in a solution, special attention being given to the diffusion of subunits via which microtubule ends can communicate with one another.

There is no certainty on whether diffusion can play a crucial behavior in dynamics situation, as it is such a fast process, especially when compared to the rates of dynamics at microtubule ends.

It might be considered why interphase and mitotic microtubules differ in the viewpoint of dynamics, growth rates, and length distribution, and account for these with altered rates of catastrophe, rescue, and nucleation. The maintenance of synchrony throughout a solution can be cleared with cooperativity among subunits at microtubule ends and signaling between them via their disassembly products [14-18]. 


\subsection{Docking simulation.}

The docking simulation applied a triangle matcher as the score function of setting methods. Some of the important force fields such as Charmm, Amber, $\mathrm{MM}+$, and $\mathrm{BIO}+$ were accomplished for refining the docking results. Data of docking explained the affinity represented and binding interaction of each compound on the microtubule components.

Tubulins in human are macromolecules which have an effect on microtubule pressure. Based on our previous methods and experience of biology information and macromolecular systems, we simulated our model theoretically [21-77]. In this work, Auto dock Tools and also iGEMDOCK software has been used, and via this method, the suitable receptors can be selected for the binding site in whole microtubules components and tubulin structures. The protein macromolecules are worked by a ligand, and iGEMDOCK can help rapidly define the appropriate binding sites. The following items have been done in docking simulation :( a), Preparing Binding Sites on the Protein Ligands. (b) Browsing and selecting the protein files. (c) Defining the binding sites kind as the bounded ligands. (d) Designing a center for the binding sites through selected ligands. (e) Setting arranging the size of the binding sites by the extended radiuses[78-82].

\section{Results and Discussion}

\subsection{Properties and quantum approach.}

This work focuses on the MT as a possible functional entity. The internal media of the MT has its own peculiar biophysical position and is largely thought to be excluded from cytoplasmic influence, except for the $2 \mathrm{~nm}^{2}$ lateral pores 1 and two $200 \mathrm{~nm}^{2}$ entrances at its ends. Several groups have expanded our view of what life is like inside MTs (Fig .4).

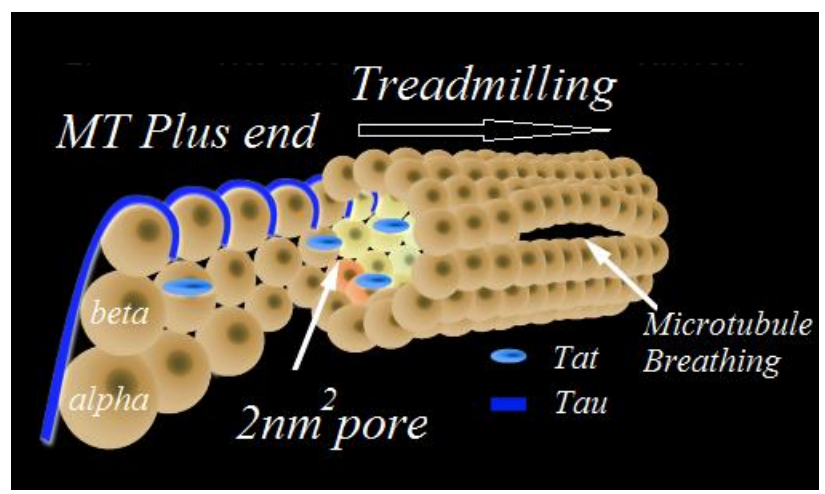

Figure 4. The entry points into the MT including TAT and tau molecules, treadmilling, $2 \mathrm{~nm} 2$ pores, a 200 nm2 open MT plus end.

A specific quantum field theoretical approach was originated to the structural constituents of brain cells, which formulated a relationship between quantum physics and memory. They emphasized that each neuron specifies a spatially distributed system with quantum mechanical degrees of freedom whose physical properties can be understood by quantum field theory.

Quantum field theory is a framework of quantum physics capable of describing fundamental processes of elementary particle physics as well as condensed matter physics. It can be suggested that memory is a quantum vacuum state that violates an original dynamical symmetry property of the full complement of the spatially-distributed quantum mechanical 
degrees of freedom in each brain cell. The introduction of quantum theory in brain science is perhaps analogous to the recent advent of the new field, quantum cosmology.

Hameroff suggested in very general terms two key concepts in understanding cytoskeletal brain activity from the point of view of quantum physics: microtubules acting as waveguides for photons and as holographic information processors. Holography is an optical phenomenon related to the interference of coherent electromagnetic waves or photons. The microtubule may be seen as a waveguide for photons, and Frohlich's theory suggests coherent excitations in microtubules. Further, the periodic lattice structure of microtubules may provide periodically arrayed 'slits' (spaces between dimers) through which photons may pass. No proof of coherent photon generation or emission necessary for optical holography has been found in microtubules or any microscopic biological structure. However, self-focused photons and self-trapped, non-thermalizing wave entities should be difficult to detect. In any case, no thorough theoretical investigation of these concepts has occurred from the standpoint of physics except the water laser (Figs.5\& 6.)

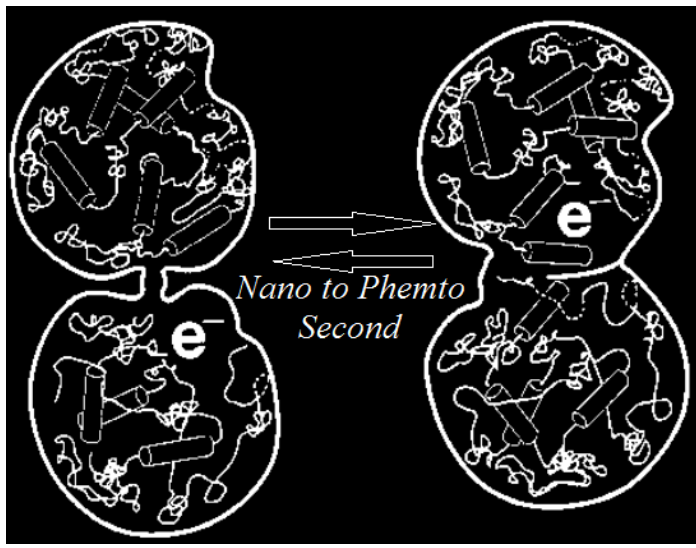

Figure 5. Alpha and beta tubulins dimer proteins have two states in which a quantum event electron mobility or quasi-particle phonon.

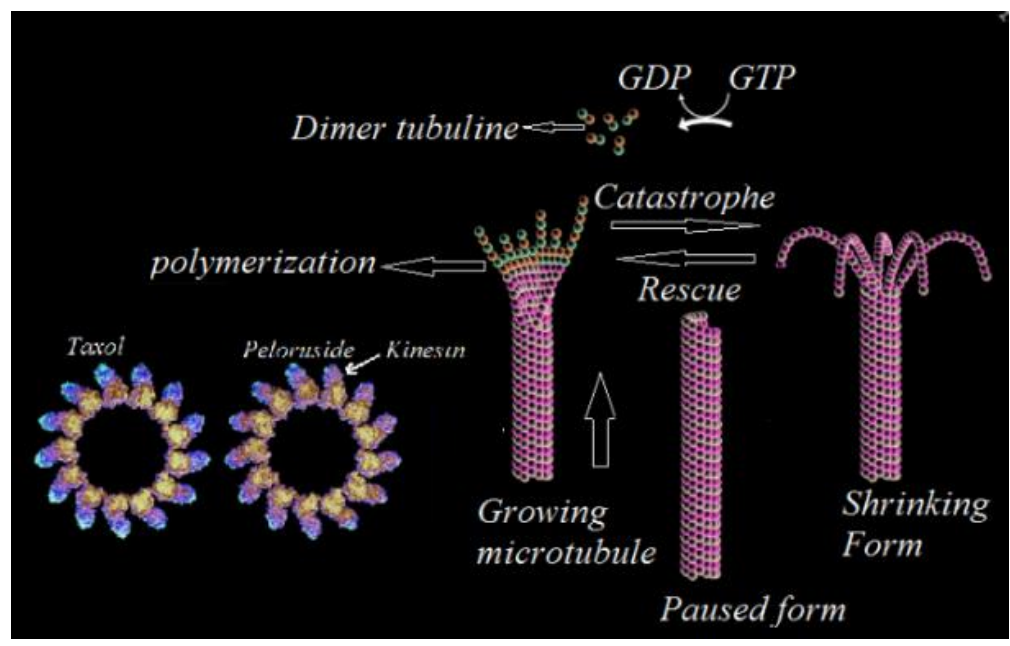

Figure 6. Taxoids ligand taxol in polymerization.

\subsection{Docking of ligands with $\alpha \beta$ - tubulin dimer.}

Molecular modeling studies simulated the amino acid residues interacting with the taxol binding site of the $\beta$-tubulin, where molecular docking of four taxoids ligand taxol (TXL) (Fig. 6 \&7) have been conducted. The results of Glide docking with 1TUB and 1JFF for the bestdocked configurations are presented in Table 1. 
Table 1. Van der Waals, Coulombic energies of the best ten docked configurations of ligands complexed within the active site of microtubules by TXL through docking and MD simulations.

\begin{tabular}{l|l|l|l|l|l} 
TXL & $\mathbf{E}_{\mathbf{v d w}}$ & $\mathbf{E}_{\text {Coulomb }}$ & Binding energy & $\mathbf{d}(\mathbf{H} \ldots \mathbf{A})(\mathbf{\AA})$ & $\begin{array}{l}\text { Angle (D- } \\
\text { H...A) }\end{array}$ \\
\hline I & -45.8 & -16.7 & -61.7 & 2.025 & 150.4 \\
\hline II & -40.8 & -15.6 & -51.9 & 2.015 & 140.5 \\
\hline III & -44.2 & -17.8 & -73.4 & 2.010 & 149.3 \\
\hline IV & -50.4 & -17.9 & -71.8 & 2.014 & 143.8 \\
\hline V & -45.8 & -16.7 & -61.7 & 2.023 & 161.9 \\
\hline VI & -46.8 & -16.3 & 55.5 & 2.025 & 153.4 \\
\hline VII & -48.9 & -16.9 & -59.7 & 2.015 & 151.8 \\
\hline VIII & -44.8 & -17.4 & -62.8 & 2.015 & 145.9 \\
\hline IX & -46.5 & -16.9 & -63.6 & 2.025 & 164.2 \\
\hline X & -45.5 & -17.9 & -60.6 & 2.015 & 154.4
\end{tabular}

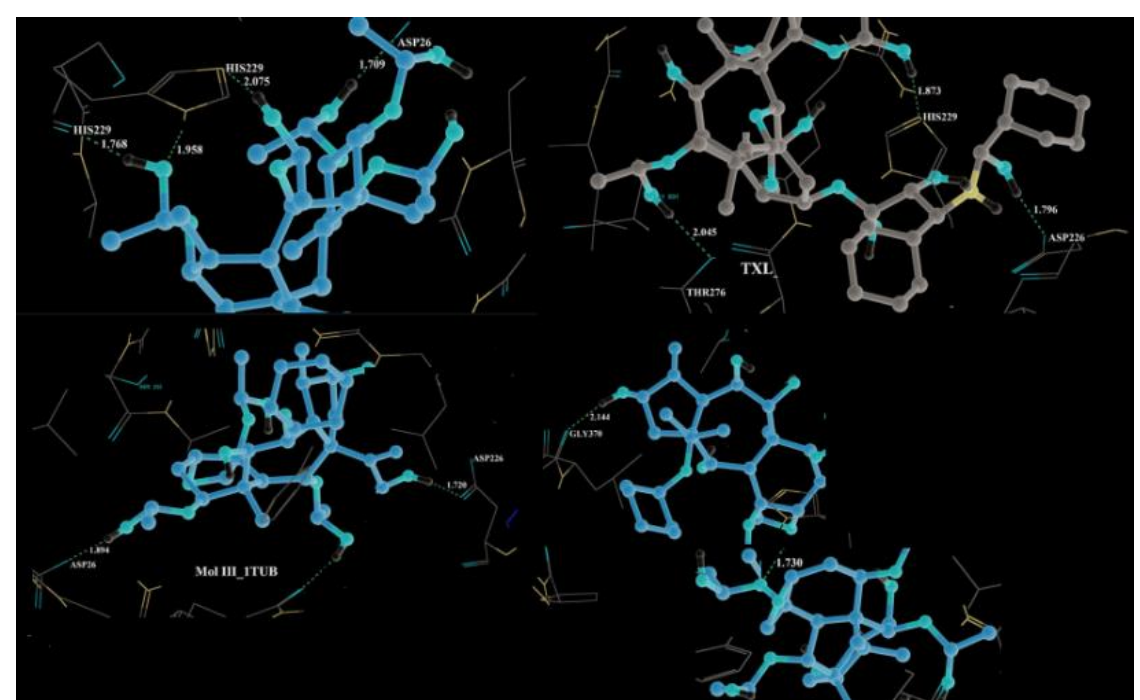

Figure 7. Hydrogen bonding in the best docking of 10 configurations of ligands with $\alpha \beta$ - tubulins dimer.

\section{Conclusions}

By this work, it has been illustrated the mechanism of microtubules depends on two systems of tubulins with stathmin curvature and taxol. The sections of two parts of the stathmin have a specific behavior for a non-bonded interaction between two tubulins and stathmin. This subunit polymerize end to end for any formation of proto-filaments similar to hollow tubes. Its dynamic instability is controlled through numerous molecules. Although the bending occurs even in the absence of destabilizing agents, the core helixes are likely of communication from the top to the bottom of the $\beta$ - subunit via a cooperative mechanism. In addition, docking studies of the TXL with tubulins give an approach concerning the binding energies and interactions within the classical taxol-binding sites of the microtubule. Results exhibit which taxol has suitable binding potential than all the other components for investigation of microtubules mechanism.

\section{Funding}

This research received no external funding.

\section{Acknowledgments}

Thanks to the Central Tehran Branch, Islamic Azad University for supporting computational software and all necessary equipment. 


\section{Conflicts of Interest}

\section{The authors declare no conflict of interest.}

\section{References}

1. Prosser, S.L.; Pelletier, L. Mitotic spindle assembly in animal cells: A fine balancing act. Nat. Rev. Mol.Cell Biol. 2017, 18, 187-201, https://doi.org/10.1038/nrm.2016.162.

2. Chaaban, S.; Brouhard, G.J. A microtubule bestiary: Structural diversity in tubulin polymers. Mol. Biol. Cell 2017, 28, 2924-2931, https://doi.org/10.1091/mbc.e16-05-0271.

3. Janke, C.; Magiera, M.M. The tubulin code and its role in controlling microtubule properties and functions. Nat. Rev. Mol. Cell Biol. 2020, 21, 307-326, https://doi.org/10.1038/s41580-020-0214-3.

4. Akhmanova, A.; Steinmetz, M.O. Microtubule minus-end regulation at a glance. J. Cell Sci. 2019, 132, https://doi.org/10.1242/jcs.227850.

5. Tovey, C.A.; Conduit, P.T. Microtubule nucleation by gamma-tubulin complexes and beyond. Essays Biochem. 2018, 62, 765-780, https://doi.org/10.1042/ebc20180028.

6. Thawani, A.; Kadzik, R.S.; Petry, S. XMAP215 is a microtubule nucleation factor that functions synergistically with the gamma-tubulin ring complex. Nat. Cell Biol. 2018, 20, 575-585, https://doi.org/10.1038/s41556-018-0091-6.

7. Luders, J. XMAP215 joins microtubule nucleation team. Nat. Cell Biol. 2018, 20, 508-510, https://doi.org/10.1038/s41556-018-0100-9.

8. Woodruff, J.B.; Ferreira Gomes, B.; Widlund, P.O.; Mahamid, J.; Honigmann, A.; Hyman, A.A. The Centrosome Is a Selective Condensate that Nucleates Microtubules by Concentrating Tubulin. Cell 2017, 169, 1066-1077.e10, https://doi.org/10.1016/j.cell.2017.05.028.

9. Vemu, A.; Atherton, J.; Spector, J.O.; Moores, C.A.; Roll-Mecak, A. Tubulin isoform composition tunes microtubule dynamics. Mol. Biol. Cell 2017, 28, 3564-3572, https://doi.org/10.1091/mbc.e17-02-0124.

10. Yates, B.; Braschi, B.; Gray, K.A.; Seal, R.L.; Tweedie, S.; Bruford, E.A. Genenames.org: The HGNC and VGNC resources in 2017. Nucleic Acids Res. 2017, 45, D619-D625, https://doi.org/10.1093/nar/gkw1033.

11. Shin, S.C.; Im, S.K.; Jang, E.H.; Jin, K.S.; Hur, E.M.; Kim, E.E. Structural and Molecular Basis for KataninMediated Severing of Glutamylated Microtubules. Cell Rep. 2019, 26, 1357-1367.e5, https://doi.org/10.1016/j.celrep.2019.01.020.

12. Guild, J.; Ginzberg, M.B.; Hueschen, C.L.; Mitchison, T.J.; Dumont, S. Increased lateral microtubule contact at the cell cortex is sufficient to drive mammalian spindle elongation. Mol. Biol. Cell 2017, 28, 1975-1983, https://doi.org/10.1091/mbc.e17-03-0171.

13. Gillard, G.; Roper, K. Control of cell shape during epithelial morphogenesis: Recent advances. Curr. Opin. Genet. Dev. 2020, 63, 1-8, https://doi.org/10.1016/j.gde.2020.01.003.

14. Dogterom, M.; Koenderink, G.H. Actin-microtubule crosstalk in cell biology. Nat. Rev. Mol. Cell Biol. 2019, 20, 38-54, https://doi.org/10.1038/s41580-018-0067-1.

15. Burute, M.; Kapitein, L.C. Cellular Logistics: Unraveling the Interplay Between Microtubule Organization and Intracellular Transport. Annu Rev. Cell Dev. Biol. 2019, 35, 29-54, https://doi.org/10.1146/annurevcellbio-100818-125149.

16. Noordstra, I.; Akhmanova, A. Linking cortical microtubule attachment and exocytosis. F1000Res 2017, 6, https://doi.org/10.12688/f1000research.10729.1.

17. Bernabe-Rubio, M.; Alonso, M.A. Routes and machinery of primary cilium biogenesis. Cell Mol. Life Sci. 2017, 74, 4077-4095, https://doi.org/10.1007/s00018-017-2570-5.

18. Spassky, N.; Meunier, A. The development and functions of multiciliated epithelia. Nat. Rev. Mol. Cell Biol.2017, 18, 423-436, https://doi.org/10.1038/nrm.2017.21.

19. Meiring, J.C.M.; Shneyer, B.I.; Akhmanova, A. Generation and regulation of microtubule network asymmetry to drive cell polarity. Curr. Opin. Cell Biol. 2020, 62, 86-95, https://doi.org/10.1016/j.ceb.2019.10.004.

20. Meaders, J.L.; Burgess, D.R. Microtubule-Based Mechanisms of Pronuclear Positioning. Cells 2020, 9 , https://dx.doi.org/10.3390\%2Fcells9020505.

21. Mollaamin, F.; Monajjemi, M. DFT outlook of solvent effect on function of nano bioorganic drugs. Physics and Chemistry of Liquids 2012, 50, 596-604, https://doi.org/10.1080/00319104.2011.646444..

22. Mollaamin, F.; Gharibe, S.; Monajjemi, M. Synthesis of various nano and micro ZnSe morphologies by using hydrothermal method. International Journal of Physical Sciences 2011, 6, 1496-1500.

23. Monajjemi M. Graphene/(h-BN)n/X-doped raphene as anode material in lithium ion batteries $(\mathrm{X}=\mathrm{Li}, \mathrm{Be}$, B AND N,). Macedonian Journal of Chemistry and Chemical Engineering 2017, 36, 101-118, http://dx.doi.org/10.20450/mjcce.2017.1134.

24. Monajjemi, M. Cell membrane causes the lipid bilayers to behave as variable capacitors: A resonance with self-induction of helical proteins. Biophysical Chemistry 2015, 207, 114-127, https://doi.org/10.1016/j.bpc.2015.10.003. 
25. Monajjemi, M. Study of CD5+ Ions and Deuterated Variants $(\mathrm{CHxD}(5-\mathrm{x})+)$ : An Artefactual Rotation. Russian Journal of Physical Chemistry A 2018, 92, 2215-2226.

26. Monajjemi, M. Liquid-phase exfoliation (LPE) of graphite towards graphene: An ab initio study. Journal of Molecular Liquids, 2017, 230, 461-472, https://doi.org/10.1016/j.molliq.2017.01.044.

27. Jalilian, H.; Monajjemi, M. Capacitor simulation including of X-doped graphene (X $=\mathrm{Li}, \mathrm{Be}, \mathrm{B})$ as two electrodes and $(\mathrm{h}-\mathrm{BN}) \mathrm{m}(\mathrm{m}=1-4)$ as the insulator. Japanese Journal of Applied Physics 2015, 54, 0851017.

28. Ardalan, T.; Ardalan, P.; Monajjemi, M. Nano theoretical study of a C 16 cluster as a novel material for vitamin C carrier. Fullerenes Nanotubes and Carbon Nanostructures 2014, 22, 687-708, https://doi.org/10.1080/1536383X.2012.717561.

29. Mahdavian, L.; Monajjemi, M.; Mangkorntong, N. Sensor response to alcohol and chemical mechanism of carbon nanotube gas sensors Fullerenes Nanotubes and Carbon Nanostructures 2009, 17, 484-495, https://doi.org/10.1080/15363830903130044.

30. Monajjemi, M.; Najafpour, J. Charge density discrepancy between NBO and QTAIM in single-wall armchair carbon nanotubes. Fullerenes Nanotubes and Carbon Nano structures 2014, 22, 575-594, https://doi.org/10.1080/1536383X.2012.702161.

31. Monajjemi, M.; Hosseini, M.S. Non bonded interaction of B16 N16 nano ring with copper cations in point of crystal fields. Journal of Computational and Theoretical Nanoscience 2013, 10, 2473-2477.

32. Monajjemi, M.; Mahdavian, L.; Mollaamin, F. Characterization of nanocrystalline silicon germanium film and nanotube in adsorption gas by Monte Carlo and Langevin dynamic simulation. Bulletin of the Chemical Society of Ethiopia 2008, 22, 277-286, https://doi.org/10.4314/bcse.v22i2.61299.

33. Lee, V.S.; Nimmanpipug, P.; Mollaamin, F.; Thanasanvorakun, S.; Monajjemi, M. Investigation of single wall carbon nanotubes electrical properties and normal mode analysis: Dielectric effects. Russian Journal of Physical Chemistry A 2009, 83, 2288-2296, https://doi.org/10.1134/S0036024409130184.

34. Mollaamin, F.; Najafpour, J.; Ghadami, S.; Akrami, M.S.; Monajjemi, M. The electromagnetic feature of B $\mathrm{N} \mathrm{H}(\mathrm{x}=0,4,8,12,16$, and 20) nano rings:Quantum theory of atoms in molecules/NMR approach. Journal of Computational and Theoretical Nanoscience 2014, 11, 1290-1298.

35. Monajjemi, M.; Mahdavian, L.; Mollaamin, F.; Honarparvar, B. Thermodynamic investigation of enolketo tautomerism for alcohol sensors based on carbon nanotubes as chemical sensors. Fullerenes Nanotubes and Carbon Nanostructures 2010, 18, 45-55, https://doi.org/10.1080/15363830903291564.

36. Monajjemi, M.; Ghiasi, R.; Seyed, S.M.A. Metal-stabilized rare tautomers: N4 metalated cytosine ( $\mathrm{M}=\mathrm{Li}$, $\mathrm{Na}, \mathrm{K}, \mathrm{Rb}$ and $\mathrm{Cs}$ ), theoretical views. Applied Organometallic Chemistry 2003, 17, 635-640, https://doi.org/10.1002/aoc.469.

37. Ilkhani, A.R.; Monajjemi, M. The pseudo Jahn-Teller effect of puckering in pentatomic unsaturated rings $\mathrm{C}$ $\mathrm{AE}, \mathrm{A}=\mathrm{N}, \mathrm{P}, \mathrm{As}, \mathrm{E}=\mathrm{H}, \mathrm{F}, \mathrm{Cl}$.Computational and Theoretical Chemistry 2015, 1074, 19-25, http://dx.doi.org/10.1016\%2Fj.comptc.2015.10.006.

38. Monajjemi, M. Non-covalent attraction of $\mathrm{B} \mathrm{N}$ and repulsion of $\mathrm{B} \mathrm{N}$ in the $\mathrm{B} \mathrm{N}$ ring: a quantum rotatory due to an external field. Theoretical Chemistry Accounts 2015, 134, 1-22, https://doi.org/10.1007/s00214-0151668-9.

39. Monajjemi, M.; Naderi, F.; Mollaamin, F.; Khaleghian, M. Drug design outlook by calculation of second virial coefficient as a nano study. Journal of the Mexican Chemical Society 2012, 56, 207-211, https://doi.org/10.29356/jmcs.v56i2.323.

40. Monajjemi, M.; Bagheri, S.; Moosavi, M.S. Symmetry breaking of B2N(-,0,+): An aspect of the electric potential and atomic charges. Molecules 2015, 20, 21636-21657, https://doi.org/10.3390/molecules201219769.

41. Monajjemi, M.; Mohammadian, N.T. S-NICS: An aromaticity criterion for nano molecules. Journal of Computational and Theoretical Nanoscience 2015, 12, 4895-4914, https://doi.org/10.1166/jctn.2015.4458.

42. Monajjemi, M.; Ketabi, S.; Hashemian, Z.M.; Amiri, A. Simulation of DNA bases in water: Comparison of the Monte Carlo algorithm with molecular mechanics force fields. Biochemistry (Moscow) 2006, 71, 1-8, https://doi.org/10.1134/s0006297906130013.

43. Monajjemi, M.; Lee, V.S.; Khaleghian, M.; Honarparvar, B.; Mollaamin, F. Theoretical Description of Electromagnetic Nonbonded Interactions of Radical, Cationic, and Anionic NH2BHNBHNH2 Inside of the B18N18 Nanoring. J. Phys. Chem C 2010, 114, 15315-15330, https://doi.org/10.1021/jp104274z.

44. Monajjemi, M.; Boggs, J.E. A New Generation of BnNn Rings as a Supplement to Boron Nitride Tubes and Cages. J. Phys. Chem. A 2013, 117, 1670-1684, http://dx.doi.org/10.1021/jp312073q.

45. Monajjemi, M. Non bonded interaction between BnNn (stator) and BN B (rotor) systems: A quantum rotation in IR region. Chemical Physics 2013, 425, 29-45, https://doi.org/10.1016/j.chemphys.2013.07.014.

46. Monajjemi, M.; Robert, W.J.; Boggs, J.E. NMR contour maps as a new parameter of carboxyl's OH groups in amino acids recognition: A reason of tRNA-amino acid conjugation. Chemical Physics 2014, 433, 1-11, https://doi.org/10.1016/j.chemphys.2014.01.017.

47. Monajjemi, M. Quantum investigation of non-bonded interaction between the B15N15 ring and BH2NBH2 (radical, cation, and anion) systems: a nano molecularmotor. Struct Chem 2012, 23, 551-580, http://dx.doi.org/10.1007/s11224-011-9895-8. 
48. Monajjemi, M. Metal-doped graphene layers composed with boron nitride-graphene as an insulator: a nanocapacitor. Journal of Molecular Modeling 2014, 20, https://doi.org/10.1007/s00894-014-2507-y.

49. Mollaamin, F.; Monajjemi, M.; Mehrzad, J. Molecular Modeling Investigation of an Anti-cancer Agent Joint to SWCNT Using Theoretical Methods. Fullerenes, Nanotubes and Carbon Nanostructures 2014, 22, 738751, https://doi.org/10.1080/1536383X.2012.731582.

50. Monajjemi, M.; Ketabi, S.; Amiri, A. Monte Carlo simulation study of melittin: protein folding and temperature ependence. Russian journal of physical chemistry 2006, 80, S55-S62, https://doi.org/10.1134/S0036024406130103.

51. Monajjemi, M.; Heshmata, M.; Haeria, H.H. QM/MM model study on properties and structure of some antibiotics in gas phase: Comparison of energy and NMR chemical shift. Biochemistry (Moscow) 2006, 71, S113-S122, https://doi.org/10.1134/S0006297906130190.

52. Monajjemi, M.; Afsharnezhad, S.; Jaafari, M.R.; Abdolahi, T.; Nikosade, A.; Monajemi, H. NMR shielding and a thermodynamic study of the effect of environmental exposure to petrochemical solvent on DPPC, an important component of lung surfactant. Russian Journal of Physical Chemistry A 2007, 81, 1956-1963, https://doi.org/10.1134/S0036024407120096.

53. Mollaamin, F.; Noei, M.; Monajjemi, M.; Rasoolzadeh, R. Nano theoretical studies of fMET-tRNA structuren in protein synthesis of prokaryotes and its comparison with the structure of fALA-tRNA. Africanjournal of microbiology research 2011, 5, 2667-2674, https://doi.org/10.5897/AJMR11.310.

54. Monajjemi, M.; Heshmat, M.; Haeri, H.H.; Kaveh, F. Theoretical study of vitamin properties from combined QM-MM methods: Comparison of chemical shifts and energy. Russian Journal of Physical Chemistry 2006, 80, 1061-1068, https://doi.org/10.1134/S0036024406070119.

55. Monajjemi, M.; Chahkandi, B. Theoretical investigation of hydrogen bonding in Watson-Crick, Hoogestein and their reversed and other models: comparison and analysis for configurations of adenine-thymine base pairs in 9 models. Journal of Molecular Structure: THEOCHEM 2005, 714, 43-60, https://doi.org/10.1016/j.theochem.2004.09.048.

56. Monajjemi, M.; Honarparvar, B.; Haeri, H.H.; Heshmat, M. An ab initio quantum chemical investigation of solvent-induced effect on 14N-NQR parameters of alanine, glycine, valine, and serine using a polarizable continuum model. Russian Journal of Physical Chemistry 2006, 80, S40-S44, https://doi.org/10.1134/S0036024406130073.

57. Monajjemi, M.; Seyed Hosseini, M. Non Bonded Interaction of B16N16 Nano Ring with Copper Cations in Point of Crystal Fields. Journal of Computational and Theoretical Nanoscience 2013, 10, 2473-2477, https://doi.org/10.1166/jctn.2013.3233.

58. Monajjemi, M.; Farahani, N.; Mollaamin, F. Thermodynamic study of solvent effects on nanostructures: phosphatidylserine and phosphatidylinositol membranes. Physics and Chemistry of Liquids 2012, 50, 161172, https://doi.org/10.1080/00319104.2010.527842.

59. Monajjemi, M.; Ahmadianarog, M. Carbon Nanotube as a Deliver for Sulforaphane in Broccoli Vegetable in Point of Nuclear Magnetic Resonance and Natural Bond Orbital Specifications. Journal of Computational and Theoretical Nanoscience 2014, 11, 1465-1471, https://doi.org/10.1166/jctn.2014.3519.

60. Monajjemi, M.; Ghiasi, R.; Ketabi, S.; Passdar, H.; Mollaamin, F. A Theoretical Study of Metal-Stabilised Rare Tautomers Stability: N4 Metalated Cytosine (M=Be2+, Mg2+, Ca2+, Sr2+ and Ba2+) in Gas Phase and Different. Journal of Chemical Research 2004, 1, 11-18, https://doi.org/10.3184/030823404323000648.

61. Monajjemi, M.; Baei, M.T.; Mollaamin, F. Quantum mechanic study of hydrogen chemisorptions on nanocluster vanadium surface. Russian Journal of Inorganic Chemistry 2008, 53, 1430-1437, https://doi.org/10.1134/S0036023608090143.

62. Mollaamin, F.; Baei, M.T.; Monajjemi, M.; Zhiani, R.; Honarparvar, B. A DFT study of hydrogen chemisorption on V (100) surfaces. Russian Journal of Physical Chemistry A, Focus on Chemistry 2008, 82, 2354-2361, https://doi.org/10.1134/S0036024408130323.

63. Monajjemi, M.; Honarparvar, B.; Nasseri, S.M.; Khaleghian, M. NQR and NMR study of hydrogen bonding interactions in anhydrous and monohydrated guanine cluster model: A computational study. Journal of Structural Chemistry 2009, 50, 67-77, https://doi.org/10.1007/s10947-009-0009-z.

64. Monajjemi, M.; Aghaie, H.; Naderi, F. Thermodynamic study of interaction of TSPP, CoTsPc, and FeTsPc with calf thymus DNA. Biochemistry (Moscow) 2007, 72, 652-657, https://doi.org/10.1134/S0006297907060089.

65. Monajjemi, M.; Heshmat, M.; Aghaei, H.; Ahmadi, R.; Zare, K. Solvent effect on 14N NMR shielding of glycine, serine, leucine, and threonine: Comparison between chemical shifts and energy versus dielectric constant. Bulletin of the Chemical Society of Ethiopia 2007, 21, 111-116, https://doi.org/10.4314/bcse.v21i1.61387.

66. Monajjemi, M.; Rajaeian, E.; Mollaamin, F.; Naderi, F.; Saki, S. Investigation of NMR shielding tensors in 1,3 dipolar cycloadditions: solvents dielectric effect. Physics and Chemistry of Liquids 2008, 46, 299-306, https://doi.org/10.1080/00319100601124369.

67. Mollaamin, F.; Varmaghani, Z.; Monajjemi, M. Dielectric effect on thermodynamic properties in vinblastine by DFT/Onsager modelling. Physics and Chemistry of Liquids 2011, 49, 318-336, https://doi.org/10.1080/00319100903456121. 
68. Monajjemi, M.; Honaparvar, B.; Khalili Hadad, B.; Ilkhani, A.; Mollaamin, F. Thermo-Chemical Investigation and NBO Analysis of Some anxileotic as Nano- Drugs. African journal of pharmacy and pharmacology 2010, 4, 521-529.

69. Monajjemi, M.; Khaleghian, M.; Mollaamin, F. Theoretical study of the intermolecular potential energy and second virial coefficient in the mixtures of $\mathrm{CH} 4$ and $\mathrm{Kr}$ gases: a comparison with experimental data. Molecular Simulation 2010, 36, 865-870, https://doi.org/10.1080/08927022.2010.489557.

70. Monajjemi, M.; Khosravi, M.; Honarparvar, B.; Mollaamin, F. Substituent and solvent effects on the structural bioactivity and anticancer characteristic of catechin as a bioactive constituent of green tea. International Journal of Quantum Chemistry 2011, 111, 2771-2777, https://doi.org/10.1002/qua.22612.

71. Tahan, A.; Monajjemi, M. Solvent dielectric effect and side chain mutation on the structural stability of Burkholderia cepacia lipase active site: a quantum mechanical/molecular mechanics study. Acta Biotheor 2011, 59, 291-312, https://doi.org/10.1007/s10441-011-9137-x.

72. Monajjemi, M.; Khaleghian, M. EPR Study of Electronic Structure of [CoF6]3-and B18N18 Nano Ring Field Effects on Octahedral Complex. Journal of Cluster Science 2011, 22, 673-692, https://doi.org/10.1007/s10876-011-0414-2.

73. Monajjemi, M.; Mollaamin, F. Molecular Modeling Study of Drug-DNA Combined to Single Walled Carbon Nanotube. Journal of Cluster Science 2012, 23, 259-272, https://doi.org/10.1007/s10876-011-0426-y.

74. Mollaamin, F.; Monajjemi, M. Fractal Dimension on Carbon Nanotube-Polymer Composite Materials Using Percolation Theory. Journal of Computational and Theoretical Nanoscience 2012, 9, 597-601, https://doi.org/10.1166/jctn.2012.2067.

75. Mahdavian, L.; Monajjemi, M. Alcohol sensors based on SWNT as chemical sensors: Monte Carlo and Langevin dynamics simulation. Microelectronics Journal 2010, 41, 142-149, https://doi.org/10.1016/j.mejo.2010.01.011.

76. Monajjemi, M.; Falahati, M.; Mollaamin, F. Computational investigation on alcohol nanosensors in combination with carbon nanotube: a Monte Carlo and ab initio simulation. Ionics 2013, 19, 155-164, https://doi.org/10.1007/s11581-012-0708-x.

77. Monajjemi, M. Molecular vibration of dopamine neurotransmitter: a relation between its normal modes and harmonic notes. Biointerface Research in Applied Chemistry, 2019, 9, 3956-3962, https://doi.org/10.33263/BRIAC93.956962.

78. Mollaamin, F.; Naiemi, M.; Monajjemi, M.; Vinblastine and vincristine as anticancer molecules stopping the tubulin dimers, Letters in applied nano bio sciences, 2019, 9, 870-874, https://doi.org/10.33263/LIANBS91.870874.

79. Kendrick, A.A.; Dickey, A.M.; Redwine, W.B.; Tran, P.T.; Vaites, L.P.; Dzieciatkowska, M.; Harper, J.W.; Reck-Peterson, S.L. Hook3 is a scaffold for the opposite-polarity microtubule-based motors cytoplasmic dynein-1 and KIF1C. J. Cell Biol. 2019, 218, 2982-3001, https://doi.org/10.1083/jcb.201812170.

80. Vuolo, L.; Stevenson, N.L.; Mukhopadhyay, A.G.; Roberts, A.J.; Stephens, D.J. Cytoplasmic dynein-2 at a glance. J. Cell Sci. 2020, 133, https://doi.org/10.1242/jcs.240614.

81. Reck-Peterson, S.L.; Redwine, W.B.; Vale, R.D.; Carter, A.P. The cytoplasmic dynein transport machinery and its many cargoes. Nat. Rev. Mol. Cell Biol. 2018, 19, 382-398, https://doi.org/10.1038/s41580-0180004-3.

82. Hertzler,J.I.; Simonovitch, S.I.; Albertson, R.M.; Weiner,A.T.; Nye, D.M.R.; Rolls, M.M.; Kinetochore proteins suppress neuronal microtubule dynamics and promote dendrite regeneration Molecular Biology of the Cell, 2020, 31, https://doi.org/10.1091/mbc.E20-04-0237-T. 